\title{
Improving adjustments for older age in pre-hospital assessment and care
}

\author{
Marius Rehn ${ }^{1,2}$
}

\begin{abstract}
Population estimates projects a significant increase in the geriatric population making elderly trauma patients more common. The geriatric trauma patients experience higher incidence of pre-existing medical conditions, impaired age-dependent physiologic reserve, use potent drugs and suffer from trauma system related shortcomings that influence outcomes. To improve adjustments for older age in pre-hospital assessment and care, several initiatives should be implemented. Decision-makers should make system revisions and introduce advanced point-of-care initiatives to improve outcome after trauma for the elderly.
\end{abstract}

\section{An ageing population}

Optimal trauma care relies on decision-makers that dynamically accommodate emergency medical services (EMS) to national trends, including demographical variations. Several population estimates indicate increased life expectancy and growth in population size. Statistics Norway projects a significant increase in population proportion above 67 years of age, pictorially calling it "the grey tsunami" (c.f. Figure 1 for demographic projections) [1]. A similar trend is seen in UK where the population is ageing and is projected to continue ageing over the next decades [2]. As a consequence, our patients are getting older making geriatric trauma more common.

\section{Extremes of age; geriatric trauma patient}

EMS providers must adjust for the changes that occur in patients at extremes of age. While children have different baseline vital signs and airway anatomy, elderly people have sensory changes that result in deviating physiological responses and manifestations of shock [3,4]. The pattern of injuries among geriatric patients is predominated by blunt trauma such as falls, motor vehicle collisions and pedestrian events [5-7]. Unsurprisingly, there is a positive correlation between increasing age and increasing incidence in preexisting medical conditions [8,9]. Pre-existing co-morbidity has been associated with an age-independent increase in

\footnotetext{
Correspondence: marius.rehn@norskluftambulanse.no

'Department of Research, Norwegian Air Ambulance Foundation, P. O. Box 94, Drøbak 1448, Norway

${ }^{2}$ Department of Anaesthesia and Intensive Care, Akershus University Hospital, Lørenskog, Norway
}

(c) 2013 Rehn; licensee BioMed Central Ltd. This is an Open Access article distributed under the terms of the Creative Commons Attribution License (http://creativecommons.org/licenses/by/2.0), which permits unrestricted use, distribution, and reproduction in any medium, provided the original work is properly cited.

\section{Death, disease, destitution}

The geriatric trauma patients are exposed to several risk factors: higher incidence of pre-existing medical conditions, impaired age-dependent physiologic reserve, potent drugs and trauma system related shortcomings. How does this influence outcome?

Kuhne and colleagues found mortality to increase at 56 years and that the increase was independent of injury

mortality after trauma. Skaga and colleagues found that pre-injury co-morbidity scored according to the American Hollis and colleagues found that pre-existing co-morbidity was associated with increased mortality when combined with low to moderate severity trauma, but not when comwith more severe trauma [12].

Further, the geriatric trauma population is a more injury. Anticoagulants increase the risk for intracranial haemorrhage and longer hospital stays following head [13,14]. Further, beta-blockers may mask tachycarfollowing injury [4].

Lastly, elderly trauma victims are exposed to age-related They also conducted a follow-up survey of the EMS ing, unfamiliarity with triage protocol, and age bias to be causal factors for the age-dependent undertriage. 


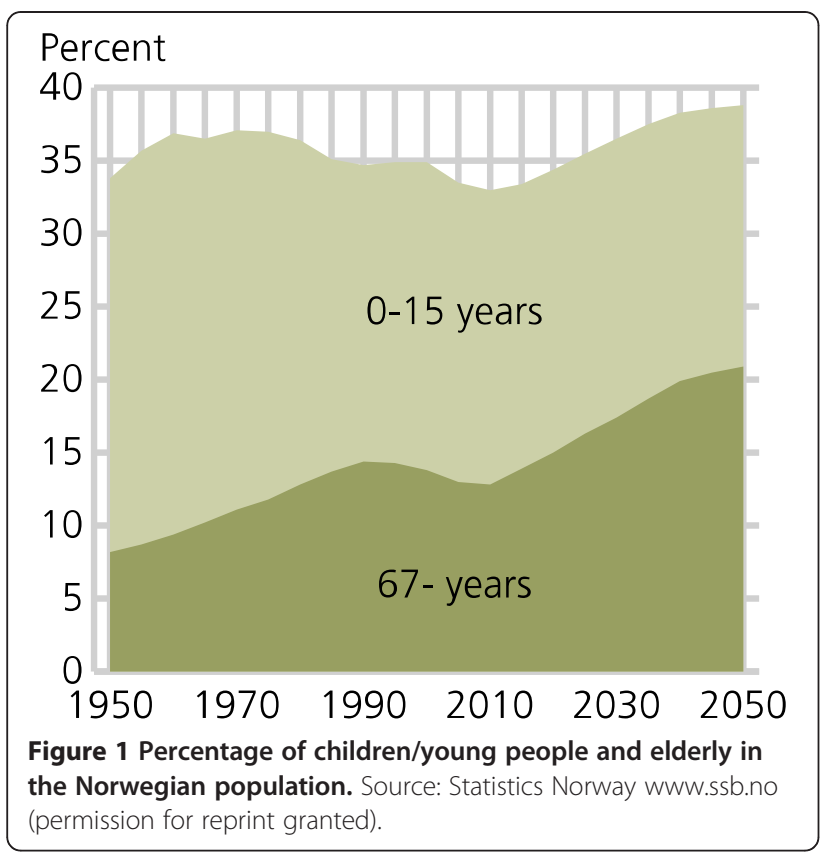

severity [16]. Søreide and colleagues found that elderly patients are more at risk for multi organ failure (MOF) and that MOF occur despite low injury severity [17]. Other studies indicate that elderly patients have higher complication rates such as infections compared to younger cohorts and that they die later in their admissions $[18,19]$. Accordingly, elderly patients have poorer outcomes and consume disproportionate amount of resources due to frequent admissions and lengthier stays at intensive care units.

\section{Improvement initiatives}

The "grey tsunami" poses a challenge to clinical decisionmakers as it creates a larger demand for already scarce prehospital resources. However, increased EMS funding alone cannot meet this challenge. Clinical leaders must launch quality improvement initiatives to increase the value of their services (c.f. List of suggested improvement initiatives) [20]. By increasing the numerator (quality) while maintaining the denominator (cost), the value will increase (c.f. Figure 2 for value equation) [21].

\section{List of suggested improvement adjustments}

- Quality improvement initiatives

- Update field triage guidelines

- Field access to national patient core record

- Field access to digital prescription system

- Termination of resuscitation rules

- Tiered trauma team activation

- Geriatric consultation service as add on to the trauma team

- Field access to arterial blood gas analyses

- Field access to radiological evaluations

\section{quality Value = --------- cost}

Figure 2 Value equation. Increase value: Increase quality and/ or decrease cost.

\section{System revisions}

Newgard and colleagues found that triage criteria loose sensitivity at the ends of age spectrum, introducing the need for separate elderly-specific criteria [22]. Heffernan and colleagues found that mortality significantly increases with systolic blood pressure (SBP) $<110 \mathrm{mmHg}$ in the geriatric patients but not until a SBP of $95 \mathrm{mmHg}$ in the younger cohorts. Chisholm and colleagues found that approximately $35 \%$ of all deaths among patients aged $\geq 65$ years were attributed to ground level falls [23]. A combined literature review and US national expert panel consensus resulted in the 2009 "Guidelines for Field Triage of Injured Patients" [24,25]. In the 2011 revision of these field triage guidelines, two criteria addressing geriatric trauma have been added: "SBP $<110$ might represent shock after age 65 years" and "Low-impact mechanisms (e.g., ground-level falls) might result in severe injury" [26]. Accordingly, decision makers should implement updated evidence-based field triage guidelines to reduce age-related mistriage.

EMS providers should establish pre-injury ASA to adjust for pre-existing medical conditions in their evaluations of geriatric trauma victims [11]. Implementing field access to a national patient core record would facilitate co-morbidity adjustments improving triage accuracy among the elderly. Further, field access to a digital prescription system would inform EMS providers of medication use that influence further management.

Wuerz and colleagues found that age has less effect on resuscitation success than other factors such as early cardio pulmonary resuscitation and advanced life support. They concluded that high age alone should not deter resuscitation attempts. However, to reduce uncertainness in end-of-life considerations in geriatric patients, EMS providers should have access to evidencebased termination of resuscitation rules [27].

A tiered approach with a reduced trauma team has proven to reduce the threshold for trauma team activation while avoiding unnecessary resource consumption [28]. Tiered trauma teams are recommended by the US National guidelines for field triage and should be considered as an improvement adjustment to accommodate 
the increasing number of elderly trauma victims [26]. Further, a geriatric consultation service as add on to the trauma team has been recommended [29]. These experts may optimize pre-existing medical conditions and manage delirium already in the initial phase of in-hospital resuscitation.

\section{Point-of care diagnostics}

Martin and colleagues found that occult hypoperfusion as determined by base deficit and lactic acid was present in $42 \%$ of geriatric patients with "normal" vital signs [30]. Field access to arterial blood gas analyses may improve the point-of-care diagnostic accuracy, conversely contributing to improved triage and treatment initiatives.

Elderly patients with impaired consciousness may prove a diagnostic challenge to EMS providers. The Norwegian Air Ambulance Foundation is currently implementing pre-hospital computer tomography units with telemedical support [31]. Such integrated solutions of advanced pointof-care diagnostics may identify traumatic brain injury or cerebral ischemia caused by either haemorrhage or infarction. Advanced point-of-care diagnostics may facilitate neuron-saving therapy already in the field. Although the feasibility of such advanced interventions remains uncertain, decision-makers should include EMS in the technological development seen in other areas of medicine. By combining low-tech system revisions with high-tech diagnostics, we may improve our adjustments for older age in pre-hospital assessment and care.

\section{Abbreviations}

EMS: Emergency Medical Services; ASA: American Society of

Anaesthesiologists; MOF: Multi Organ Failure; SBP: Systolic Blood Pressure.

Received: 5 November 2012 Accepted: 21 January 2013

Published: 23 January 2013

\section{References}

1. Statistics Norway: An ageing society. 2012. http://www.ssb.no/norge_en/ bef_en.pdf.

2. Office for National Statistics: Population ageing in the United Kingdom, its constituent countries and the European Union. 2012. http://www.ons.gov.uk/ ons/dcp171776_258607.pdf.

3. Mock C, Lormand J, Goosen J, Joshipura M, Peden M: Guidelines for essential trauma care. Geneva: World Health Organization; 2004.

4. Demetriades D, Sava J, Alo K, Newton E, Velmahos GC, Murray JA, Belzberg $\mathrm{H}$, Asensio JA, Berne TV: Old age as a criterion for trauma team activation. J Trauma 2001, 51:754-756. discussion 756-757.

5. Coronado VG, Thomas KE, Sattin RW, Johnson RL: The CDC traumatic brain injury surveillance system: characteristics of persons aged 65 years and older hospitalized with a TBI. J Head Trauma Rehabil 2005, 20:215-228.

6. Grossman MD, Miller D, Scaff DW, Arcona S: When is an elder old? Effect of preexisting conditions on mortality in geriatric trauma. J Trauma 2002, 52:242-246.

7. Oreskovich MR, Howard JD, Copass MK, Carrico CJ: Geriatric trauma: injury patterns and outcome. J Trauma 1984, 24:565-572.

8. Ford ES, Giles WH, Dietz WH: Prevalence of the metabolic syndrome among US adults: findings from the third National Health and Nutrition Examination Survey. JAMA 2002, 287:356-359.
9. Battistella FD, Din AM, Perez L: Trauma patients 75 years and older: longterm follow-up results justify aggressive management. J Trauma 1998, 44:618-623. discussion 623

10. ASA Physical Status Classification System. American Society of Anesthesiologists: 2012. http://www.asahq.org/clinical/ physicalstatus.htm.

11. Skaga NO, Eken T, Sovik S, Jones JM, Steen PA: Pre-injury ASA physical status classification is an independent predictor of mortality after trauma. J Trauma 2007, 63:972-978.

12. Hollis S, Lecky F, Yates DW, Woodford M: The effect of pre-existing medical conditions and age on mortality after injury. J Trauma 2006, 61:1255-1260

13. Brewer ES, Reznikov B, Liberman RF, Baker RA, Rosenblatt MS, David CA, Flacke S: Incidence and predictors of intracranial hemorrhage after minor head trauma in patients taking anticoagulant and antiplatelet medication. J Trauma 2011, 70:E1-E5.

14. Wong DK, Lurie F, Wong LL: The effects of clopidogrel on elderly traumatic brain injured patients. J Trauma 2008, 65:1303-1308.

15. Chang DC, Bass RR, Cornwell EE, Mackenzie EJ: Undertriage of elderly trauma patients to state-designated trauma centers. Arch Surg 2008 143:776-781. discussion 782.

16. Kuhne CA, Ruchholtz S, Kaiser GM, Nast-Kolb D: Mortality in severely injured elderly trauma patients-when does age become a risk factor? World J Surg 2005, 29:1476-1482.

17. Soreide K, Kruger AJ, Vardal AL, Ellingsen CL, Soreide E, Lossius HM: Epidemiology and contemporary patterns of trauma deaths: changing place, similar pace, older face. World J Surg 2007, 31:2092-2103.

18. Schiller WR, Knox R, Chleborad W: A five-year experience with severe injuries in elderly patients. Accid Anal Prev 1995, 27:167-174.

19. Giannoudis PV, Harwood PJ, Court-Brown C, Pape HC: Severe and multiple trauma in older patients; incidence and mortality. Injury 2009, 40:362-367.

20. Davidoff F, Batalden P, Stevens D, Ogrinc G, Mooney S: Publication guidelines for quality improvement in health care: evolution of the SQUIRE project. Qual Saf Health Care 2008, 17(Suppl 1):i3-i9.

21. ACS-COT: Trauma performance improvement reference manual. Chicago: American College of Surgeons; 2002.

22. Newgard CD, Zive D, Holmes JF, Bulger EM, Staudenmayer $K$, Liao M, Rea T, Hsia RY, Wang NE, Fleischman R, et al: A multisite assessment of the American College of Surgeons Committee on Trauma field triage decision scheme for identifying seriously injured children and adults. J Am Coll Surg 2011, 213:709-721.

23. Chisholm KM, Harruff RC: Elderly deaths due to ground-level falls. Am J Forensic Med Pathol 2010, 31:350-354.

24. Sasser SM, Hunt RC, Sullivent EE, Wald MM, Mitchko J, Jurkovich GJ, Henry MC, Salomone JP, Wang SC, Galli RL, et al: Guidelines for field triage of injured patients. Recommendations of the national expert panel on field triage. MMWR Recomm Rep 2009, 58:1-35.

25. Lerner EB: Studies evaluating current field triage: 1966-2005. Prehosp Emerg Care 2006, 10:303-306.

26. Sasser SM, Hunt RC, Faul M, Sugerman D, Pearson WS, Dulski T, Wald MM, Jurkovich GJ, Newgard CD, Lerner EB: Guidelines for field triage of injured patients: recommendations of the National Expert Panel on Field Triage, 2011. MMWR Recomm Rep 2012, 61:1-20.

27. Nolan JP, Soar J, Zideman DA, Biarent D, Bossaert LL, Deakin C, Koster RW, Wyllie J, Bottiger B: European resuscitation council guidelines for resuscitation 2010. Resuscitation 2010, 81:1219-1276.

28. Rehn M, Lossius HM, Tjosevik KE, Vetrhus M, Ostebo O, Eken T: Efficacy of a two-tiered trauma team activation protocol in a Norwegian trauma centre. Br J Surg 2012, 99:199-208.

29. Richmond TS, Kauder D, Strumpf N, Meredith T: Characteristics and outcomes of serious traumatic injury in older adults. J Am Geriatr Soc 2002, 50:215-222.

30. Martin JT, Alkhoury F, O'Connor JA, Kyriakides TC, Bonadies JA: 'Normal' vital signs belie occult hypoperfusion in geriatric trauma patients. Am Surg 2010, 76:65-69.

31. Lossius HM, Lund CG: Pre-hospital treatment of stroke - time is brain. Tidsskr Nor Laegeforen 2012, 132:1848-1849.

doi:10.1186/1757-7241-21-4

Cite this article as: Rehn: Improving adjustments for older age in prehospital assessment and care. Scandinavian Journal of Trauma,

Resuscitation and Emergency Medicine 2013 21:4. 